\title{
Valproic Acid Exposure during Early Postnatal Gliogenesis Leads to Autistic-like Behaviors in Rats
}

\author{
Tamanna Jahan Mony ${ }^{1}$, Jae Won Lee ${ }^{1,2}$, Cheryl Dreyfus ${ }^{3}$, Emanuel DiCicco-Bloom ${ }^{3,4}$, Hee Jae Lee ${ }^{1}$ \\ ${ }^{1}$ Department of Pharmacology, Kangwon National University School of Medicine, Chuncheon, ${ }^{2}$ Natural Medicine Research Center, Korea \\ Research Institute of Bioscience and Biotechnology, Cheongju, Korea, ${ }^{3}$ Department of Neuroscience and Cell Biology, Rutgers Robert \\ Wood Johnson Medical School, Rutgers, the State University of New Jersey, Piscataway, ${ }^{4}$ Department of Pediatrics, Rutgers Robert Wood \\ Johnson Medical School, Rutgers, the State University of New Jersey, New Brunswick, NJ, USA
}

\begin{abstract}
Objective: We reported that postnatal exposure of rats to valproic acid (VPA) stimulated proliferation of glial precursors during cortical gliogenesis. However, there are no reports whether enhanced postnatal gliogenesis affects behaviors related to neuropsychiatric disorders.

Methods: After VPA treatment during the postnatal day (PND) 2 to PND 4, four behavioral test, such as open field locomotor test, elevated plus maze test, three-chamber social interaction test, and passive avoidance test, were performed at PND 21 or 22 .

Results: VPA treated rats showed significant hyperactive behavior in the open field locomotor test $(p<0.05)$. Moreover, the velocity of movement in the VPA group was increased by $69.5 \%(p<0.01)$. In the elevated plus maze test, VPA exposed rats expressed significantly lower percentage of time spent on and of entries into open arms more than the control group $(p<0.05)$. Also, both sociability and social preference indices with strangers in the three-chamber social interaction test were significantly lower in the VPA exposed rats $(p<0.05)$.

Conclusion: Our results suggest that altered glial cell development is another locus at which pathogenetic factors can operate to contribute to the neurodevelopmental disorder.
\end{abstract}

KEY WORDS: Valproic acid; Postnatal gliogenesis; Social behavior; Anxiety; Autistic disorder.

\section{INTRODUCTION}

Valproic acid (VPA) is a well-known anticonvulsant used as a therapeutic agent. VPA is commonly used to treatment of seizures, anxiety, bipolar disorder, epilepsy and prevent migraines. ${ }^{1-4)}$ Due to its adverse teratogenic effects, it is prescribed for pregnant women with significant cautions. ${ }^{5,6)}$ The VPA-induced animal model is based on the assumption that VPA administration during the early stages of fetal development interrupts the neurodevelopmental process eventually leading to behavior abnormalities that are similar to autism. ${ }^{7,8)}$ Both pre- and postnatal exposures to VPA in rodents have been found to elicited autism like neurobehavioral defects that may cor-

\footnotetext{
Received: April 28, 2016 / Revised: May 11, 2016

Accepted: May 14, 2016

Address for correspondence: Hee Jae Lee, PhD

Department of Pharmacology, Kangwon National University School

of Medicine, 1 Gangwondaehak-gil, Chuncheon 24341, Korea

Tel: +82-33-250-8852, Fax: +82-33-242-7571

E-mail: heejaelee@kangwon.ac.kr
}

respond to the motor and cognitive deficits, observed in human cases of autism. .-11) $^{-1}$

An important process that contributes to neurodevelopmental disorder is the generation of glial cells or gliogenesis. Glia is the predominant cells in the brain. ${ }^{12)}$ Astrocytes are the most numerous cells in the mammalian brain, and an increasing ratio of glia to neurons is associated with the progressive development of higher brain function. ${ }^{13)}$ Glia plays an important role in brain metabolism, synaptic neurotransmission, synaptic plasticity and communication with neurons. ${ }^{14,15}$ Early postnatal maturing astrocytes also promote synaptogenesis and the balance between synapse stabilization and elimination. ${ }^{16-18)}$ During early developmental stages the dysfunction of astrocytes has been found to relate to psychiatric disorder including Rett syndrome, fragile X mental retardation, Alexander's disease, epilepsy and autistic spectrum disorders. ${ }^{12)}$ Thus, the determination of the effects of VPA on astrocytogenesis in the developing brain might provide insight into mechanisms that to contribute to autism-related behaviors.

() This is an Open-Access article distributed under the terms of the Creative Commons Attribution Non-Commercial License (http://creativecommons.org/licenses/by-nc/4.0) which permits unrestricted non-commercial use, distribution, and reproduction in any medium, provided the original work is properly cited. 
Previously, to define the effects of VPA on astrocytogenesis, we designed our study to administer VPA during the first postnatal week when glial precursor proliferation is the predominant event in the developing brain. ${ }^{19)}$ Early postnatal exposure to VPA between postnatal day (PND) 2 to PND 4 in the rats represents the third trimester of human gestation. We found that this brief VPA exposure leads to increased astrocytogenesis and juvenile glia due to increased precursor cell proliferation. ${ }^{19)}$ In the current study, we examine potential effects of enhanced astrocytogenesis on behaviors related to neurodevelopmental disorders.

\section{METHODS}

\section{Animals}

All of the animal experiments were conducted by the animal care guidelines of the National Institutes of Health (NIH) and the Korean Academy of Medical Sciences. This study was approved by the Institutional Animal Care and Use Committee of Kangwon National University (KW-130313-1). Fourteen pregnant Sprague-Dawley rats were obtained from Taconic (Seongnam, Korea) and maintained on a standard 12-hour light-dark cycle, at ambient temperature $\left(22^{\circ} \mathrm{C} \pm 2^{\circ} \mathrm{C}\right)$ and humidity $(55 \% \pm 5 \%)$ with liberated access to food pellets and water. Rats were checked every morning for delivery, if newborn pups were seen, that day was considered as PND 0. Sixty-four male pups in total were used in our behavioral studies. Behavior studies were performed during the daytime between 10.00 AM to 4.00 PM. Before the behavioral studies, animals were habituated to the place of the experiment for a minimum of one hour before the start of the test. The open field locomotion test was carried out on PND 21, followed by the elevated plus maze on PND 22. Social interaction test and passive avoidance test were performed independently on PND 22; the same animal has never been used for the different behavioral study.

\section{Valproic Acid (VPA) Treatment}

The procedure used here was the same as previously described by Lee et al. ${ }^{19)}$ Briefly, pups were injected subcutaneously in the dorsal neck region with $300 \mathrm{mg} / \mathrm{kg}$ VPA (Sigma, St. Lois, MO, USA) twice daily on PND 2 and PND 3, and once on PND 4. An equal amount of saline was injected into each rat in the control group.

All animal treatment, including administration of drug and euthanasia, were carried out by the principle of Laboratory Animal Care (NIH publication N0-85-23, revised 1985).

\section{Open Field Locomotors Test}

On PND 21, exploratory activity in a novel environment was assessed in an open field box $(90 \times 90 \mathrm{~cm})$ divided into 81 squares of identical size $(10 \times 10 \mathrm{~cm})$ enclosed by continuous black plexiglas. Rats were introduced into the center area of the arena and habituated for five minutes and left to move for recording actual behavior. Rat behavior was measured continuously and recorded with a video camera placed over the structure. The total distance moved (total bar crossing in units) in the whole arena, and the velocity of crossing bar was measured. Controls $(n=15)$ and VPA treated group $(n=10)$ were used for this analysis.

\section{Elevated Plus-Maze}

On PND 22, an elevated plus-maze test (open arm, $50 \times 10 \times 40 \mathrm{~cm}$; close arm, $50 \times 10 \times 40 \mathrm{~cm}$; height, $40 \mathrm{~cm}$ ) was conducted according to the previously reported procedures. $^{20)}$ Briefly, subjects were placed in the center of the maze, facing an open arm, and allowed to explore the maze. The total entries into open arms and the ratio of stay time in open arm versus total spent time were measured. Controls $(n=15)$ and VPA treated group $(n=10)$ were used for this analysis.

\section{Social Interaction Test}

A three-chamber social interaction test was performed on PND 22 as previously described. ${ }^{21)}$ Subjects were habituated in the middle compartment with doors closed for five minutes before the test. In the first session, subjects were placed in the middle of the compartment. Then strange animal (same age and strain) was placed into the wired cage of either the left or the right compartment. This new rat represented the stranger zone 1 while the other wired cage remained empty as an empty zone. The rats were left for a 10-minute sociability test. The time spent in the stranger zone 1 with interaction to the new rat versus time spent in the empty zone were measured and are expressed as a ratio. The social preference test was conducted for another 10 minutes period after termination of the sociability test. Another new animal was introduced into the wired cage in place of the empty zone and was consider as stranger zone 2 . The same parameters were measured to define preference of the subjected animal to choose interaction with the familiar animal or the novel one. The tracking of movement was recorded using the video recorder. Sociability and social preference indices were calculated followed by the formulas described early. ${ }^{21)}$ Controls $(n=10)$ and VPA treated group $(n=9)$ were used for this analysis. 


\section{Passive Avoidance Test}

On PND 22, the memory of animals was tested by a passive avoidance test. The apparatus (Gemini; SD Instruments, San Diego, CA, USA) consisted of a shuttle box equipped with a door to restrict access between equal-sized $(25 \times 25 \times 35 \mathrm{~cm})$ illuminated and dark compartments. In the training trial, rats were individually placed in the illuminated compartment for 180 seconds and allowed to enter the dark compartment through an opened door between the two compartments. After some time, the animal spontaneously entered the dark compartment. The door was shut one second after the crossing, and the rat was given an unavoidable scrambled electric foot shock ( $1 \mathrm{~mA} / 2$ seconds) through the grid floor of the dark compartment. Then 24 hours later, the same procedure was repeated without an electric foot shock. A latency of entry greater than 300 seconds was a criterion for successful learning. Controls $(\mathrm{n}=10)$ and VPA treated group $(\mathrm{n}=10)$ were used for this analysis.

\section{Statistical Analysis}

All results have been expressed as the mean \pm standard error of the mean for each group. The statistical differences between two groups were determined by the Student's $t$-test. $p$ values less than 0.05 were considered to indicate statistically significant differences.

\section{RESULTS}

\section{Early Postnatal VPA Treatment Induces Hyperactive Behavior}

On PND 21 we performed an open field test with both a control and VPA treated groups for locomotor activity.
The units of total bar crossing in the whole arena and velocity of crossing bar were measured for 20 minutes. In the open field test, VPA treated rats showed a 52\% increase in the total number of bars crossed, suggesting hyperactive behavior $(p<0.05$; Fig. 1A). Moreover, the velocity of crossing bar ( $\mathrm{cm} / \mathrm{second}$ ) was also significantly increased by $59 \%$ in VPA treated group as compared to the control group ( $p<0.01$; Fig. 1B), indicating that hyperactive activity followed VPA early postnatal exposure to VPA in rats.

\section{Early Postnatal VPA Treatment Induced Abnormal Anxiety Behavior}

An elevated plus-maze test was performed to assess anxiety-related behavior. On PND 22, a control and VPA treated groups were used for this analysis. The ratio of the open arm entries to total arm entries and the ratio of the spent time in the open arm were measured for this analysis. Each session lasted for eight minutes. The number of entries into the open arm (Fig. 2A) and the time spent in the open arm (Fig. 2B) were both reduced in the VPA exposed animals than the control group $(p<0.05)$. These results suggest that early postnatal VPA exposure shows an abnormal anxiety behavior.

\section{Early Postnatal VPA Treatment Induces Deficits in Social Behaviors}

To evaluate social behavior, we performed the threechamber social interaction tests on PND 22. Social interaction was measured as the time subject rat spent with an unfamiliar conspecific place under the wired cage and included running towards, sniffing, grooming, mounting and crawling over the wired cage.

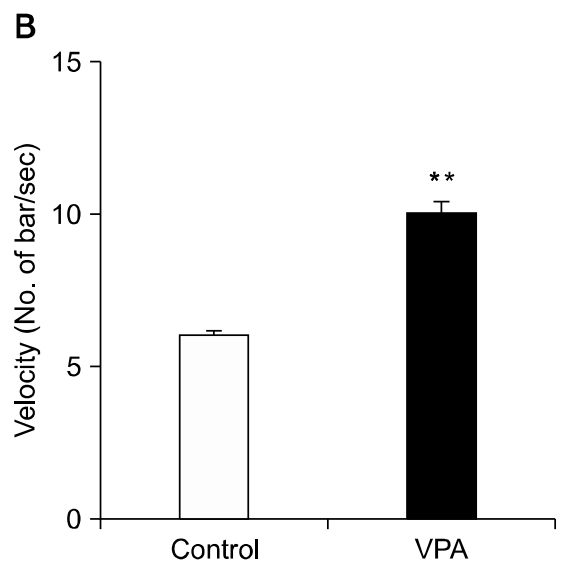

Fig. 1. Postnatal valproic acid (VPA) treatment induced hyperactivity in Sprague-Dawley rats. An open field test was performed on postnatal day 21. (A) A total number of crossing bar (units). (B) The velocity of crossing bar. Controls: $n=15$; VPA treated group: $n=10$. Data are expressed as mean \pm standard error of the mean. The asterisks indicate significant differences $\left({ }^{* *} p<0.01\right)$. 
A

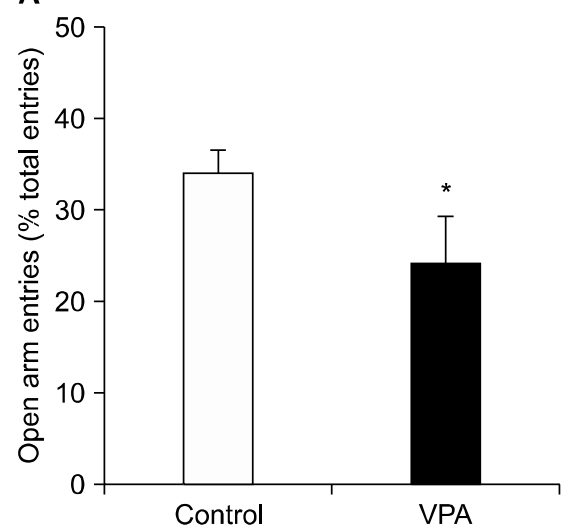

B

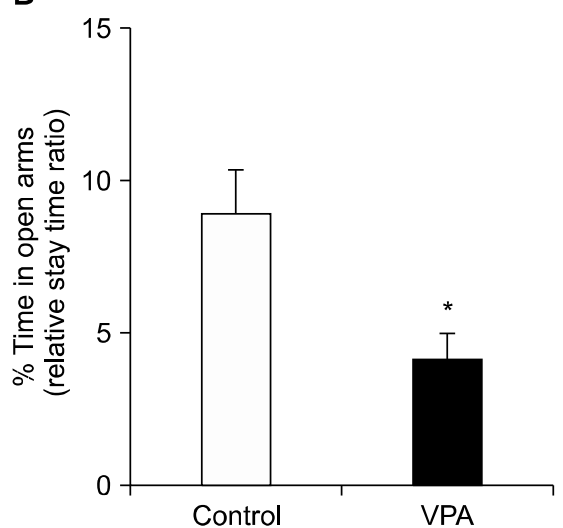

Fig. 2. Effects of postnatal valproic acid (VPA) treatment on anxiolytic activity. The elevated plus-maze test was performed on postnatal day 22. (A) The ratio of the open arm entries to total arm entries. (B) The ratio of spent time in open arms divided by total time. Controls: $\mathrm{n}=15$; VPA treated group: $\mathrm{n}=10$.

Data are expressed as mean \pm standard error of the mean.

The asterisks indicate significant differences $\left({ }^{*} p<0.05\right)$.

A

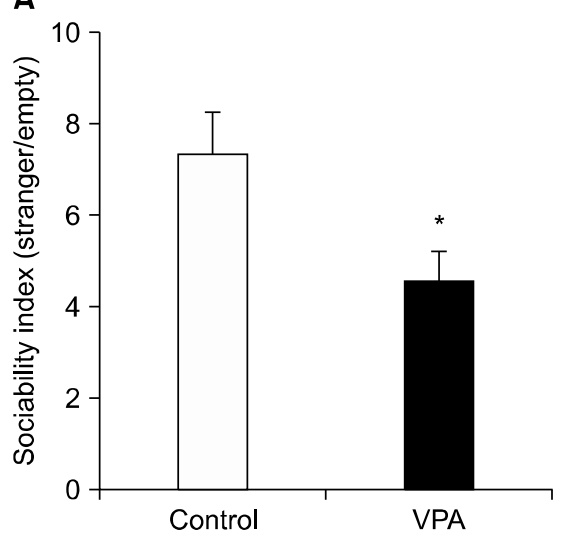

B

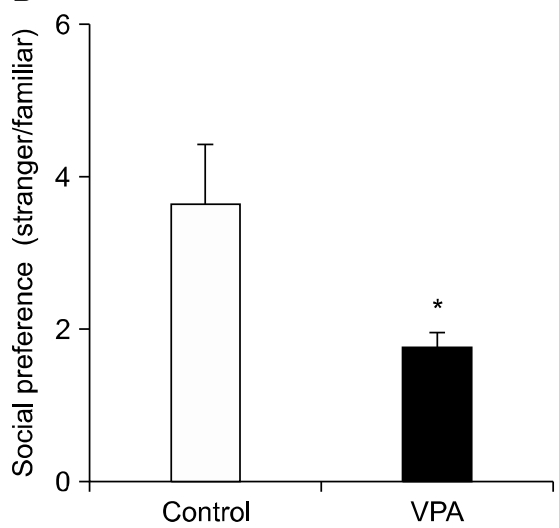

Fig. 3. Effects of postnatal valproic acid (VPA) treatment on sociability index and social preference index on the three-chamber social behavioral test. (A) Sociability index. (B) Social preference index. Controls: $n=10$; VPA treated group: $n=9$.

Data are expressed as mean \pm standard error of the mean.

The asterisks indicate significant differences $\left({ }^{*} p<0.05\right)$.

In the first session, we measured the sociability index and in the second session, we measured social preferences index between the novel and familiar rats. Each session was performed for 10 minutes. In the sociability test, the VPA treated rats showed significantly lower interaction times, reduced by $45 \%$, with conspecific rat $(p<0.05$; Fig. 3A). Furthermore, the VPA treated rats also spent significantly more time with the familiar rat and less time with the stranger $(p<0.05$; Fig. 3B). Both of these behavioral measures suggest social interaction deficits following early postnatal VPA treatment.

\section{Early Postnatal VPA Treatment Elicits No Difference in Learning and Memory Retention}

To evaluate the effect of early VPA treatment on learning and memory (avoidance learning), we performed a passive avoidance test on PND 22 (Fig. 4). No significant differences were found between the control and VPA treated groups, suggesting that early postnatal VPA treatment has no effect on this measure of learning and memory.

\section{DISCUSSION}

We reported that the appropriate concentration of VPA elicited the proliferation of astrocyte precursors both in vitro and in vivo. ${ }^{19)}$ Briefly, VPA exposure elicited dose-de- 


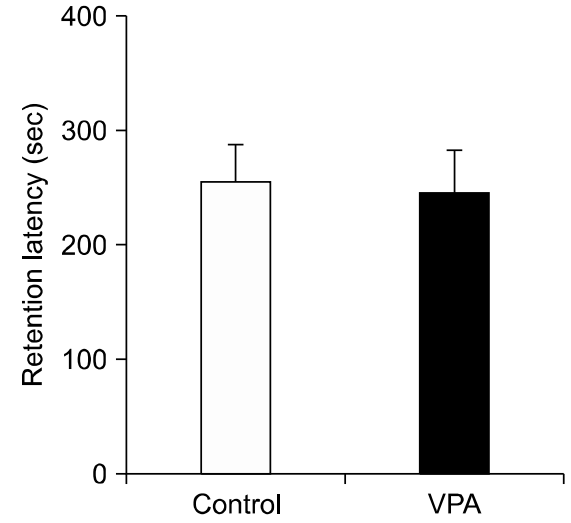

Fig. 4. Effects of postnatal valproic acid (VPA) treatment on retention latency in passive avoidance test. Controls: $n=10$; VPA treated group: $n=10$.

Data are expressed as mean \pm standard error of the mean.

pendent and biphasic effects on DNA synthesis and proliferation on primary glial precursor culture. Also, 300 $\mathrm{mg} / \mathrm{kg}$ of VPA exposure from PND 2 to 4 increased both DNA synthesis and immature astrocyte proliferation in the PND 4 frontal cortex in vivo. Furthermore, that increased glial precursor proliferation at PND 4 led to increasing astrocytes in the PND 21 frontal cortex. Because prenatal (E 12.5 to $\mathrm{E} 13$ period) exposure to VPA is a well-known animal model of autism spectrum disorder (ASD) in rats, ${ }^{22-24)}$ we confirmed whether increased number of astrocytes in the prefrontal cortex by early postnatal exposure to VPA effects autistic-like behaviors.

In the present study, we found that marked decreases in social interaction and social preference in postnatal VPA exposed rats. These results are consistent with those results in prenatal VPA exposed offspring. ${ }^{21,25)}$ Also, when VPA was exposed to PND 2 to 4, it elicited increased anxiety and hyperactivity behavior in the adolescent rats, consistent with those results of prenatal VPA exposed rats. ${ }^{9,26)}$ Based on behavioral criteria to diagnose ASD, such as persistent social communication and interaction deficit, and consistent results on prenatal VPA exposure animal model, we suggest that our new postnatal VPA exposure animal model also be an animal model of ASD. Interestingly, administration of VPA not only on PND 2 to 4 but also on PND 14 elicits increased locomotor activity in a novel environment, decreased social behavior, increased anxiety in an elevated plus maze, retardation in water maze performance. ${ }^{10,27,28)}$ Furthermore, administration of VPA at PND 14 induces sensitivity to pain, and loss of motor skill development. ${ }^{29)}$ Taken together, early postnatal exposure to VPA also may lead to neurodevelopmental deficits and induce autistic-like behaviors as prenatal VPA ex- posed offspring.

Why does early postnatal VPA exposure lead to neurodevelopmental deficits and induce autistic-like behaviors? One possibility is that increased number of astrocyte in the frontal cortex may alter the establishment of appropriate neuron-glial circuit formation that affects functional deficits. It is known that astrocytes play emerging roles in neural circuit development by controlling synapse formation, maturation, function, and elimination. ${ }^{30)}$ If abundant of astrocytes exist during glial-neural circuit formation, a surplus astrocytes may disturb normal neural circuit formation that could contribute to the pathogenesis of neurodevelopmental disorders. ${ }^{31)}$ Interestingly, the expression level of GFAP, a major astrocytic marker, and connexin 43, a major component protein in astrocytic gap junction, is increased in the superior frontal cortex of postmortem brain tissues from ASD patients. ${ }^{32,33)}$ Moreover, recent reports indicated that the number of astrocytes is three times increased, and the number of astrocytic branch processes, total branching length and cell body size is reduced in the frontal cortex of postmortem brain tissues from ASP patients. ${ }^{34)}$ Results from our previous study ${ }^{19)}$ showed that marked increases in astrocyte number and level of GFAP expression in the PND 21 frontal cortex after postnatal VPA treatment. Although it remains to determine whether early postnatal VPA exposure can alter glial plasticity, the complex behavioral phenotypes observed in ASD may be elicited by abnormal astrogenesis in the frontal lobe.

Alternatively, it is also possible that suppressed the formation of GABAergic synapses ${ }^{35)}$ and increased glutamatergic differentiation $^{36)}$ through histone deacetylase inhibition by VPA treatment, may be a potential cause of the behavioral deficits observed in ASD. Recently, it has emerged that disturbance of the excitatory/inhibitory balance is supposed mechanism responsible for the pathophysiology of ASD. ${ }^{37)}$ Notably, astrocytes release multiple signal molecules to regulate glutamatergic synapse formation. ${ }^{38)}$ If a surplus astrocytes release abnormal amounts of signal molecules, increased astrocytes could affect excitatory/inhibitory imbalance in early postnatal VPA exposed rats. Further studies will be needed to discriminate what kind of signal molecules from astrocytes elicits excitatory/inhibitory imbalance in the frontal cortex after early postnatal VPA treatment.

In conclusion, we previously suggest that VPA treatment in the early postnatal period (PND 2 to 4 ) has an effect on cortical gliogenesis during brain development. ${ }^{19)}$ This alteration in postnatal development may induce re- 
duced social interaction and hyperactivity behavior at adolescent. We, therefore, suggest that this new VPA exposure animal model may provide a new translational model of ASD.

\section{Acknowledgments}

This work was supported by grants from Kangwon National University (2011) and NICHD (HD23315-22). The authors have no conflict of interests.

\section{REFERENCES}

1. Bond DJ, Lam RW, Yatham LN. Divalproex sodium versus placebo in the treatment of acute bipolar depression: a systematic review and meta-analysis. J Affect Disord 2010; 124:228-234.

2. Kim KC, Kim P, Go HS, Choi CS, Yang SI, Cheong JH, et al. The critical period of valproate exposure to induce autistic symptoms in Sprague-Dawley rats. Toxicol Lett 2011;201:137-142.

3. Lovell BV, Marmura MJ. Valproate semisodium ER for migraine and cluster headache prophylaxis. Expert Opin Drug Metab Toxicol 2010;6:495-504.

4. Mackey C. The anticonvulsants market. Nat Rev Drug Discov 2010;9:265-266.

5. Koren G, Nava-Ocampo AA, Moretti ME, Sussman R, Nulman I. Major malformations with valproic acid. Can Fam Physician 2006;52:441-442, 444, 447.

6. Ornoy A. Valproic acid in pregnancy: how much are we endangering the embryo and fetus? Reprod Toxicol 2009; 28:1-10.

7. Chauhan A, Chauhan V. Oxidative stress in autism. Pathophysiology 2006;13:171-181.

8. Söğüt S, Zoroğlu SS, Ozyurt H, Yilmaz HR, Ozuğurlu F, Sivasli E, et al. Changes in nitric oxide levels and antioxidant enzyme activities may have a role in the pathophysiological mechanisms involved in autism. Clin Chim Acta 2003;331:111-117.

9. Schneider T, Przewłocki R. Behavioral alterations in rats prenatally exposed to valproic acid: animal model of autism. Neuropsychopharmacology 2005;30:80-89.

10. Wagner GC, Reuhl KR, Cheh M, McRae P, Halladay AK. $A$ new neurobehavioral model of autism in mice: pre-and postnatal exposure to sodium valproate. J Autism Dev Disord 2006;36:779-793.

11. Yochum CL, Dowling P, Reuhl KR, Wagner GC, Ming X. $V P A$-induced apoptosis and behavioral deficits in neonatal mice. Brain Res 2008;1203:126-132.

12. Molofsky AV, Krencik R, Ullian EM, Tsai HH, Deneen B, Richardson WD, et al. Astrocytes and disease: a neurodevelopmental perspective. Genes Dev 2012;26:891-907.

13. Nedergaard M. Direct signaling from astrocytes to neurons in cultures of mammalian brain cells. Science 1994;263: 1768-1771.

14. Rajkowska G, Miguel-Hidalgo JJ. Gliogenesis and glial pathology in depression. CNS Neurol Disord Drug Targets 2007;6:219-233.

15. Wang DD, Bordey A. The astrocyte odyssey. Prog Neurobiol 2008;86:342-367.

16. Araque A, Parpura V, Sanzgiri RP, Haydon PG. Tripartite synapses: glia, the unacknowledged partner. Trends Neurosci 1999;22:208-215.
17. Parpura V, Haydon PG. Physiological astrocytic calcium levels stimulate glutamate release to modulate adjacent neurons. Proc Natl Acad Sci U S A 2000;97:8629-8634.

18. Pfrieger FW. Roles of glial cells in synapse development. Cell Mol Life Sci 2009;66:2037-2047.

19. Lee HJ, Dreyfus C, DiCicco-Bloom E. Valproic acid stimulates proliferation of glial precursors during cortical gliogenesis in developing rat. Dev Neurobiol 2015. doi: 10.1002/dneu.22359. [Epub ahead of print]

20. Pellow S, File SE. Anxiolytic and anxiogenic drug effects on exploratory activity in an elevated plus-maze: a novel test of anxiety in the rat. Pharmacol Biochem Behav 1986;24: 525-529.

21. Kim JW, Seung H, Kwon KJ, Ko MJ, Lee EJ, Oh HA, et al. Subchronic treatment of donepezil rescues impaired social, hyperactive, and stereotypic behavior in valproic acid-induced animal model of autism. PLoS One 2014;9:e104927.

22. Arndt TL, Stodgell CJ, Rodier PM. The teratology of autism. Int J Dev Neurosci 2005;23:189-199.

23. Miyazaki K, Narita N, Narita M. Maternal administration of thalidomide or valproic acid causes abnormal serotonergic neurons in the offspring: implication for pathogenesis of autism. Int J Dev Neurosci 2005;23:287-297.

24. Stodgell CJ, Ingram JL, O'Bara M, Tisdale BK, Nau H, Rodier PM. Induction of the homeotic gene Hoxal through valproic acid's teratogenic mechanism of action. Neurotoxicol Teratol 2006;28:617-624.

25. Bambini-Junior V, Rodrigues L, Behr GA, Moreira JC, Riesgo R, Gottfried C. Animal model of autism induced by prenatal exposure to valproate: behavioral changes and liver parameters. Brain Res 2011;1408:8-16.

26. Al-Amin MM, Rahman MM, Khan FR, Zaman F, Mahmud Reza H. Astaxanthin improves behavioral disorder and oxidative stress in prenatal valproic acid-induced mice model of autism. Behav Brain Res 2015;286:112-121.

27. Chomiak T, Karnik V, Block E, Hu B. Altering the trajectory of early postnatal cortical development can lead to structural and behavioural features of autism. BMC Neurosci 2010;11:102.

28. Reynolds S, Millette A, Devine DP. Sensory and motor characterization in the postnatal valproate rat model of autism. Dev Neurosci 2012;34:258-267.

29. Pragnya B, Kameshwari JS, Veeresh B. Ameliorating effect of piperine on behavioral abnormalities and oxidative markers in sodium valproate induced autism in $B A L B / C$ mice. Behav Brain Res 2014;270:86-94.

30. Clarke LE, Barres BA. Emerging roles of astrocytes in neural circuit development. Nat Rev Neurosci 2013;14: 311-321.

31. Sloan SA, Barres BA. Mechanisms of astrocyte development and their contributions to neurodevelopmental disorders. Curr Opin Neurobiol 2014;27:75-81.

32. Laurence JA, Fatemi SH. Glial fibrillary acidic protein is elevated in superior frontal, parietal and cerebellar cortices of autistic subjects. Cerebellum 2005;4:206-210.

33. Fatemi SH, Folsom TD, Reutiman TJ, Lee S. Expression of astrocytic markers aquaporin 4 and connexin 43 is altered in brains of subjects with autism. Synapse 2008;62:501-507.

34. Cao F, Yin A, Wen G, Sheikh AM, Tauqeer Z, Malik M, et al. Alteration of astrocytes and Wnt/ $\beta$-catenin signaling in the frontal cortex of autistic subjects. J Neuroinflammation 2012;9:223.

35. Kumamaru E, Egashira Y, Takenaka R, Takamori S. Valproic acid selectively suppresses the formation of inhibitory synapses in cultured cortical neurons. Neurosci Lett 2014; 
569:142-147.

36. Kim KC, Lee DK, Go HS, Kim P, Choi CS, Kim JW, et al. Pax6-dependent cortical glutamatergic neuronal differentiation regulates autism-like behavior in prenatally valproic acid-exposed rat offspring. Mol Neurobiol 2014;49:512-528.
37. Mabunga DF, Gonzales EL, Kim JW, Kim KC, Shin CY. Exploring the validity of valproic acid animal model of autism. Exp Neurobiol 2015;24:285-300.

38. Allen NJ. Astrocyte regulation of synaptic behavior. Annu Rev Cell Dev Biol 2014;30:439-463. 